\title{
An Evaluation on the Relation of Organizational Commitment and Knowledge Sharing A Case Study: Trade Ministry Rahbar Informatics Service Co
}

\author{
Hamideh Assari \\ State Management M.A (Information System Management branch), Allame Tabatabaee University, Instructor of \\ Khansar Payam-e-Noor University, Golpayegan Payam-e-Noor University and Golpayegan Payam Non-profit University \\ Email: parisaassari@yahoo.com
}

Vajihe Safi

State Management M.A (Information System Management branch), Allame Tabatabaee University. Instructor of Najaf Abad Azad University. Emai: vajihe_safi@yahoo.com

Mojgan Assari

Industry Management M.A (System Management and Efficiency branch), Sharif Industrial University Email: mozhgan.assari@gmail.com

\section{Doi:10.5901/mjss.2016.v7n5s1p111}

\begin{abstract}
The present study has tried to evaluate the relation between the organizational commitment and knowledge sharing. In order to evaluate the research hypothesis, a sample of 110 managers, authorities and experts of Rahbar Informatics Service Company were selected. Then the research questionnaire with the reliability of $91 \%$ was distributed among the statistical samples. After collecting the data, they were abridged, classified and entered into the SPSS Software. The results revealed that all the research hypothesis are approved and the results of evaluating the research hypotheses showed that there is a meaningful relation between the organizational commitment and knowledge sharing $(P \leq 0.05)$. Finally practical suggestions will be provided for the Rahbar Informatics Service Company. It is hoped that the results of the present study can be applied to improve the staffs' behaviors and functions in sharing their knowledge and finally improve the organizational function.
\end{abstract}

Keywords: Knowledge management; knowledge sharing; organizational commitment, interactive commitment; relation commitment, assimilation commitment; Rahbar Informatics Service Company.

\section{Introduction}

Today knowledge is considered as a precious and strategic asset for the organizations and providing products and services with economical and proper quality is difficult and sometimes impossible without true and proper management and applying these valuable resources (Han, 2007). As the knowledge and information is diversely spread through the organizations with high volume and quantity, the organizations should be aware that what the common knowledge set of the organization is, how they should be managed and how they should be applied and shared to have the highest efficiency. Knowledge sharing is a key issue in the knowledge management which is of a great importance for the organizations as it enables them to improve their skills and competency and keep their competitive advantage $\left(\mathrm{Wu}^{1}\right.$, 2009). Many researchers like Nonaka and Takiochi consider knowledge sharing as a prerequisite for technology development and generating new products. On the other hand, the influential factors in increasing the staffs' motivation for knowledge sharing have always been a question (Holste \& J.Scott, 2003). One of the main goals of the managers to apply knowledge sharing in the organizations is to improve the knowledge sharing among the organizational members to provide competitive advantage. Efficient knowledge sharing among the members of the organization leads to the cost reduction and guarantees the distribution of the best working methods in the organization and it enables the organization to solve their problems, as well (Nonaka, 1995).

Knowledge sharing includes some issues like the style of sharing the present knowledge and transferring it to the 
proper and needed place, how to transfer the knowledge in such a way that it can be available and accessible in the organization, how to transfer the knowledge from the personal level to the group ones, and finally the organizational knowledge level (Afrazeh, 2005).

In an organization with the culture of sharing knowledge, the staffs share their ideas and insights with each others. That is due to the fact that they consider it as a natural process not as an obligation. Therefore this motivation should be generated among the organizational staffs to share their knowledge without the fear of losing their position and/or job (Yang \& Wan, 2004).

While knowledge sharing is one of the biggest concerns of the organizational managers that generate competitive advantage in the knowledge economy, many companies have not tried hard to plan proper methods to efficiently encourage the staffs to share their knowledge (Wu et al.). Determining those factors affecting on the staffs' motivation for sharing the knowledge is of a great importance and organizations should focus on it. As we can see, organizational leaders annually spend millions of dollars to maintain and improve the knowledge technology infrastructures with the hope of facilitating the efficient transferring of the knowledge .

Story and Quintas (2001) believe that generating trust, motivation and commitment in the staffs is one of the greatest issues in managing the staffs' knowledge (Hislop, 2003).

$\operatorname{Lin}^{2}$ (2007) has evaluated the influential factors on knowledge sharing in the organizations. In his research, he has expressed one of the influential factors on knowledge sharing as the inter-department interactions and "trust and commitment are the cores of the achievements in inter-department interactions. Commitment includes an interaction based on loyalty, fairness, and organizational high understanding". As the organizational commitment is necessary to improve the process of knowledge sharing in the organizations, if the staffs feel that by sharing their knowledge they may lose their position or job, they'll avoid sharing their knowledge with the others (Holste \& J.Scott,2003). Organizational commitment is also important as the staffs with higher organizational ranks and positions may leave the organization with less probability, and they are more stimulated and more motivated to provide volunteer efforts, and generally are more willing to share their knowledge (Hislop, 2003).

Scarbro and Carter (2000) have expressed that a set of the best efforts of the human resource management facilitate the knowledge sharing which they can be generated or improved. It's worth noting that based on this view point, these set of actions are applicable and functional for all the organizations. This insight is exactly related to organizational commitment and it is discussed that the organizations can guarantee that level of commitment, capability and knowledge sharing capacity which leads to the achievements through perusing and following the best model.

Robertson and Omaley Hammersly (2000) have found in their research that there is a positive insight to knowledge sharing among the staffs. They also have explained that the employees are in high level of job satisfaction and they are more committed to their organization. Therefore high level of organizational commitment is related to the staffs' positive insight to knowledge sharing.

On the basis of the significance of the organizational commitment and the importance of these issues in facilitating the knowledge sharing trend, more focus and research is found to be needed about this issue. Based on the researches, the highest benefit of the knowledge management for the organizations is in knowledge sharing and transformation. This knowledge sharing and distribution has its own challenges, though. In Iran, the organizations have bureaucratic hierarchy which makes this knowledge sharing more difficult. "Most of the staffs are not willing to share their knowledge with the others, as the power is based on the knowledge and having more knowledge can promote the individuals in the office hierarchies". On the other hand there is a need toward improving the organizational commitment among the staffs because any related researches have not been performed in the aforementioned company and the relation of these two variables with knowledge sharing has not been reviewed in the given organization.

It's worth mentioning that this company is concerned to research activities and the related issues more than the other companies or similar organizations and it has tried hard to resolve the barriers and problems in the field of knowledge sharing and distribution. The researchers hope to have a positive impact on this company through removing the implicit and clear problems and barriers of knowledge sharing and facilitating this issue by improving the level of organizational commitment among the staffs and they try to attract the higher authorities and mangers' attention to the significance of the key points in facilitating the knowledge sharing among the organizational staffs.

\section{Theoretical Principles}

In Oxford dictionary, the commitment is expressed as the obligation that limits discretion. Like the other organizational

$2 \operatorname{Lin}$ 
behaviors, organizational commitment is defined with various meanings. The most common method of treating the organizational management is considering that as an emotional dependency to the organizations. Based on this method, the ones with the highest commitment take their identity through the organization, participates in the organization, combine with it and enjoy their membership in that organization (Sarooghi, 1996).

Based on Lotanz and Shaw (1992), the definition of organizational commitment as an insight is:

- $\quad$ strong willingness to remain in the organization

- willingness to hardworking for the organization

- strong belief into accepting the values and goals (Iraqi, 1998).

The common point of the provided definitions is that commitment is an emotional state which determines the relation of the person with the organization and it implicitly includes staying or leaving that organization (Sarooghi, 1996).

The present research has applied Balfour and Kessler's three-part model to measure the staffs' organizational commitment that has the following dimensions:

a) Assimilation commitment: it is stated as the amount of the staffs' harmony with the goals and values of the organizations

b) Affiliation commitment: it is originated through feeling of having attachment and dependency to the organization

c) Interactive commitment: it is based on attachment to the organization and it increases when the organization determines the staffs' participation, signifies the importance of the staffs and provides support and encouragement for the staffs (Balfour \& Wechsler, 1996).

\subsection{Knowledge sharing}

"Sharing the knowledge includes issues like sharing the present knowledge and distributing it to the proper and needed location and how to transfer it in such a way that it can be available and accessible in the organization. It implies how to transfer the knowledge from the personal level to the group ones, and finally the organization knowledge is one of the issues in the instructions of the knowledge management" (Afrazeh, 2005).

Hidden (implicit) knowledge: it is defined as a mental and personal knowledge which is not easy to express, transfer, share and formulize. Hidden knowledge is not easy to codify and transformable to the rules and instructions. It is achieved through mimicking and close interaction with those ones who are the owners of the knowledge like a learner and an instructor (Nonaka, 1995).

Clear (explicit) knowledge: it is a knowledge which can be codified and developed through some signs like the letters and numbers, and in text, sound, photo, software, data center, etc formats. When the explicit knowledge is demonstrated, it can take the form of the reports, research articles, catalogues, speeches, lectures, etc. (Holste \& J.Scott, 2003).

\section{Review of Literature}

Hoof and Ridler (2004) have performed a research based on measuring the relation between the organizational commitment and knowledge sharing. In this study the authors have hypothesized that organizational commitment and relation atmosphere are the factors of development or underdevelopment of sharing the knowledge in the organizations and groups.

The result of a research by Kabrera et al. (2006) revealed that organizational commitment, as an independent variable, has a positive influence on the staffs' insight toward sharing the knowledge. It also showed this issue that in order to achieve the effects of the organizational commitment, the insight of the staffs toward sharing the knowledge should be developed with the help of human resource initiatives. They found that people with higher levels of internalizing the commitment (Oriley \& Chatman's model) are more willing to share their knowledge with the others.

Lin (2003) has evaluated the affecting factors on sharing the knowledge in the organizations. In his research, he has examined three categories of factors as intra-department relation, the features of organizational structure, and organizational culture. In the category of the features of organizational structure, authenticity, complexity and concentration are dealt with. In the intra-department relation category, trust and commitment are the cores of the achievements of the interactions in the departments. Commitment includes an interaction based on the affection, loyalty, fairness, and organizational high understanding, while trust is the desire of one of the members of the organization to the others' beliefs and it is measured by the others' competence in measuring the commitments.

The study by Hislap (2003) revealed that there is an interesting relation between the staffs' commitment level to 
their organization and their treatment and behavior style in innovation and knowledge management. The degrees of the commitment affect on the staffs' willingness or unwillingness to share the others with their knowledge.

As Story and Quintas (2001) state, the development of the staffs' trust, motivation and commitment reveals one of the main factors related to the workers' knowledge management. Employees with higher degrees of commitment tend less to leave their job and have more motivation and look for visual efforts, and accordingly are more willing to share their knowledge with the organization.

Khatamian Far (2007) has reviewed the status of knowledge sharing, infrastructures, field and methods of sharing the knowledge. The results of his study reveal that generally the infrastructure of knowledge sharing in the organization of the libraries is better. Beyond this, related to motivating and deterrent factors of knowledge sharing in this organizations, it was found that in the organizational and personal factors affecting on knowledge sharing, the lack of enough time to participate in knowledge sharing initiatives was a hindrance.

Mortazavi (2008) has reviewed in his research the three organizational structures (including the complexity, authenticity, and concentration), organizational culture (including bureaucratic culture, innovative and creative culture, and supportive culture), and the interaction between the departments as the most important human factors like trust and commitment that affect on knowledge sharing.

Yosefi et al. (2010) have reviewed the role of the organizational commitment in knowledge sharing. In this research it is tried to review the relation of the staffs' organizational commitment and knowledge sharing. The results of this study showed that high levels of organizational commitment are related to the staffs' positive insights to knowledge sharing. Staffs with more commitment to their organization are more cooperative and active in the knowledge sharing initiatives.

\section{Research Hypothetical Pattern}

In this chapter the ideas, opinions and models of the authors and researchers were studied and evaluated in different fields of intra-personal trust, organizational commitment and knowledge sharing in the organizations based on which finally the conceptual model of the study was developed. Balfour and Kessler model (1996) was applied to design the organizational commitment model. They believe that the organizational commitment has these three dimensions: assimilation commitment, affiliation commitment, and interactive commitment. In relation to the concept of the knowledge sharing with patterning the Helset model (2003), the two dimensions of knowledge sharing (implicit and explicit) were based, and finally the conceptual model of the study was designed.

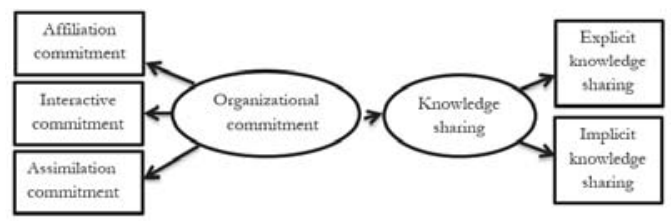

Figure 1. Research Conceptual Pattern

\section{Research Hypotheses}

\subsection{Main hypothesis}

There is a relation between the organizational commitment and knowledge sharing in Rahbar Informatics Service Company.

\subsection{Subsidiary hypotheses}

$\mathrm{H} 1$. There is a relation between the assimilation commitment and knowledge sharing in Rahbar Informatics Service Company.

$\mathrm{H} 2$. There is a relation between the affiliation commitment and knowledge sharing in Rahbar Informatics Service Company.

$\mathrm{H} 3$. There is a relation between the interactive commitment and knowledge sharing in Rahbar Informatics Service Company. 


\section{Research Method}

Based on the relation of the organizational commitment and knowledge sharing, and describing the status of the factors and as this research is performed in a real organization, an evaluative-descriptive research method was applied. As the given organization can apply the results of the present study in its future plans, it is a functional and/or practical one, as well.

Sampling method and research statistical population

The statistical population of the present study includes 155 logistic and operational staffs of Rahbar Informatics Service Company (a Trade Ministry attaché). The random sampling method is applied, as well. The following formula was applied to give the number of the samples:

$$
\mathrm{n}=\frac{\mathrm{NZ} \alpha / 2^{2} \mathrm{P}(1-\mathrm{P})}{\varepsilon^{2}(\mathrm{~N}-1)+\mathrm{Z} \alpha / 2^{2} \mathrm{P}(1-\mathrm{P})}
$$

where:

$\mathrm{N}$ : the amount of the statistical population; $n$ : the amount of the required sample; $\mathrm{Z}$ : the amount of the standard variable (given as 1.96 from the related table for the reliability of $95 \%$ ); $\varepsilon_{1}$ : the amount of the researcher's error in the evaluations which is usually $0.01-0.1$ and here is taken as $0.05 ; P(1-p)$ : quality feature variance under the evaluation which is indefinitely applied here in the maximum of $0.05^{*} 0.05=0.25$.

Based on the above formula and based on the number of the statistical population, 110 ones were selected as the research statistical sample.

\section{Data Collection Tools}

In the present study, library studies for the review of the literature were followed by questionnaires for data collection. The related questionnaires were applied to evaluate the relation of the organizational commitment and knowledge sharing. Balfour and Kessler questionnaire of organizational commitment was applied to evaluate the staffs' organizational commitment. Because of is repeatability in various studies and with its reliability and validity, this is considered as a standard questionnaire. The experts have approved its validity, as well. Helset (2003) questionnaire is also applied for knowledge sharing which was standardized in his research. To measure the validity of the research tools, 30 questionnaires were distributed between the statistical populations. After collecting the questionnaires, their alphaKronbach was measured with SPSS Software which it was given as 0.91 . It reveals that this questionnaire is reliable, or it has the proper validity.

\section{Data Analysis}

\subsection{Main hypothesis:}

"There is a relation between the organizational commitment and knowledge sharing in Rahbar Informatics Service Company".

Table 1. Correlation coefficient between the organizational commitment and knowledge sharing

\begin{tabular}{|l|c|c|c|}
\hline Variables & Correlation coefficient & Meaningfulness level & No. of respondents (n) \\
\hline organizational commitment with knowledge sharing & 0.252 & 0.034 & 110 \\
\hline
\end{tabular}

*The relation is meaningful in the level of $95 \%$.

The results of the correlation coefficient measurement between the two variables of the organizational commitment and knowledge sharing reveal that among 110 respondents of the study, the correlation coefficient with the meaningfulness level of $\alpha=0.252$ is measured. As this is less than the expected meaningfulness level of the study $(\alpha=0.05)$, there would be enough reason to reject the zero hypothesis. Therefore, $\mathrm{H}_{0}$ is rejected and $\mathrm{H}_{1}$ is approved. It can be concluded that 'there is a positive and meaningful relation between the organizational commitment and knowledge sharing'.

\subsection{Subsidiary hypothesis 1}

There is a relation between the assimilation commitment and knowledge sharing in Rahbar Informatics Service Company. 
Table 2. Correlation coefficient between the assimilation commitment and knowledge sharing

\begin{tabular}{|l|c|c|c|}
\hline Variables & Correlation coefficient & Meaningfulness level & No. of respondents (n) \\
\hline assimilation commitment with knowledge sharing & 0.253 & 0.030 & 110 \\
\hline
\end{tabular}

*The relation is meaningful in the level of $95 \%$.

The results of the correlation coefficient measurement between the two variables of the assimilation commitment and knowledge sharing reveal that among 110 respondents of the study, the correlation coefficient with the meaningfulness level of $a=0.030$ is measured as $R=0.253$. As this is less than the expected meaningfulness level of the study $(a=0.05)$, there would be enough reason to reject the zero hypothesis. Therefore, $\mathrm{H}_{0}$ is rejected and $\mathrm{H}_{1}$ is approved. It can be concluded that 'there is a positive and meaningful relation between the assimilation commitment and knowledge sharing'.

\subsection{Subsidiary hypothesis 2:}

"There is a relation between the affiliation commitment and knowledge sharing in Rahbar Informatics Service Company".

Table 3. Correlation coefficient between the affiliation commitment and knowledge sharing

\begin{tabular}{|l|c|c|c|}
\hline Variables & Correlation coefficient & Meaningfulness level & No. of respondents (n) \\
\hline affiliation commitment with knowledge sharing & 0.241 & 0.031 & 110 \\
\hline
\end{tabular}

*The relation is meaningful in the level of $95 \%$.

The results of the correlation coefficient measurement between the two variables of the affiliation commitment and knowledge sharing reveal that among 110 respondents of the study, the correlation coefficient with the meaningfulness level of $\alpha=0.030$ is measured as $R=0.241$. As this is less than the expected meaningfulness level of the study ( $\alpha=0.05)$, there would be enough reason to reject the zero hypothesis. Therefore, $\mathrm{H}_{0}$ is rejected and $\mathrm{H}_{1}$ is approved. It can be concluded that 'there is a positive and meaningful relation between the affiliation commitment and knowledge sharing'.

\subsection{Subsidiary hypothesis 3:}

"There is a relation between the interactive commitment and knowledge sharing in Rahbar Informatics Service Company".

Table 4. Correlation coefficient between the interactive commitment and knowledge sharing

\begin{tabular}{|l|c|c|c|}
\hline Variables & Correlation coefficient & Meaningfulness level & No. of respondents (n) \\
\hline interactive commitment with knowledge sharing & 0.151 & 0.491 & 110 \\
\hline
\end{tabular}

The results of the correlation coefficient measurement between the two variables of the interactive commitment and knowledge sharing reveal that among 110 respondents of the study, the correlation coefficient with the meaningfulness level of $\alpha=0.491$ is measured as $R=0.151$. As this is less than the expected meaningfulness level of the study $(\alpha=0.05)$, there would be enough reason to reject the zero hypothesis. Therefore, $\mathrm{H}_{0}$ is rejected and $\mathrm{H}_{1}$ is approved. It can be concluded that 'there is a positive and meaningful relation between the interactive commitment and knowledge sharing'.

\section{Conclusion and Suggestions}

Based on the findings of this research and the previous studies, the biggest benefit of the organizations from knowledge management is in the knowledge transfer and sharing. Today the organizations have concluded that they should focus more on what they know than the ones they have. On the other hand, in this communication age which is followed by huge advancements in the fields of information and communication, the organizations require more organized facilities to manage and organize data, information and knowledge. Today having huge amounts of knowledge and information is not beneficial, but related classified and in-time information can be supportive for the organizations. Then determining those factors affecting on the individuals' tendency to share their knowledge is of a great importance and the organizations should focus well on them. Based on the performed measurements and tests, it was found that there is a relative strong 
correlation between the organizational commitment and knowledge sharing, as the staffs in higher levels and positions of the organizations leave less the organization, and they are more motivated, tend more for voluntary efforts and generally are more eager to share their knowledge in the organization.

Based on the provided results of this study, the following functional suggestions will be given to Rahbar Informatics Service Company. It is hoped that the results of the study can be applied to improve the employees and staffs' behaviors and functions in the field of knowledge sharing and therefore, improve the organizational functions and operations.

As there is a positive and meaningful relation between the staffs' organizational commitment and knowledge sharing, it can be suggested that the organizational managers should try hard to generate a sense of organizational affiliation and commitment. The managers should maintain and develop this staffs' commitment to the organization, as this commitment attaches the staffs to the organization and the individuals will be more eager to share their knowledge with the others. Organizational commitment has serious potential effects on the functions and operations of the organizations and it can be an important preventive factor for the organizational efficiency. Then its ignorance is harmful for the organization and leads to many losses for the organization. Through measuring the organizational commitment and the level of its influence on the staffs' behaviors like work absence, work quit, work shift, etc. , proper information will be provided for the managers for their decision making, especially the human resource managers.

As believe into the organizations goals and tendency to membership and hardworking to attain the organizational goals have an important role in motivating the staffs to share their knowledge with the others, the managers are suggested to have a clear and explicit expressions of their organization's values to focus on and find out the extent of the working staffs' respect and value for the organizational ones. Then they can try to make these working staffs' values close to the organizational ones, because this accompaniment and congruence of the working staffs' values in the organization is an important factor to generate and develop the organizational commitment of the individuals to the organization. In this way, the individuals feel more assimilated with the organization and tend more to share their knowledge with the others.

Based on the general organizational behavior, one of the important affiliation commitment foundations is generating the sense of attachment to the organization through social relations which is developed through team working. Therefore organizational managers are suggested to have an organizational atmosphere in such a way that it increases the sense of cooperation and team working in the staffs and provides sincere relations between the organizational members in order to have higher levels of staffs' organizational commitment and affiliation commitment. It is in these situations that the staffs tend more to share their knowledge with their coworkers and colleagues.

Interactive commitment is based on the interchangeability of the individuals' attachment to the organization; that is, based on the bonuses and consideration each person receives from the organization. Then the attachment and affiliation to the organization increases. Therefore, based on the findings of this hypothesis, the managers of Rahbar Informatics Service Company are suggested to support and encourage their staffs and employees through encouragement and appreciation the staffs' functions and operations, bonus, proper evaluation of the staffs and determining those staffs with higher functionality, and finding out the cooperation of the active staffs in the organization. With these initiatives the staffs will feel that they are important for the managers and their attachment to the organization will increase. Therefore they desire to be more efficient to attain the organizational goals and then the knowledge sharing increases.

\section{References}

Afrazeh,A. (2005). Knowledge Management: Concepts, Models, Measurement and Running. Amir Kabir Industrial University Publication, Tehran.

Balfour, L. Danny., and Wechsler, Barton (1996). Organizational Commitment, Public Productivity \& Management Review, Vol. 19, No.3, pp. 256-277.

Balfour, L. Danny., \& Wechsler, Barton (1991). Commitment, Performance, and Productivity in Public Organization, Public Productivity \& Management Review, Vol. 14, No.4, pp. 355-367.

Han, Brent \& Anantatmula, Vi al (2007) "Knowledge sharing in large IT organizations: a Case study" Journal of information and knowledge management system, 37, 421-439.

Hislop,D.(2003),Linking Human Resource Management and Knowledge Management via commitment : A Review Research Agenta ,Employee Relation, Vol. 25,pp .182-202.

Hoof, Bart van den, Ridder, Jan A. de(2004). Knowledge sharing in context: The influence of organizational commitment, communication climate and CMC use on knowledge sharing, Journal of knowledge management, $8,117$.

Holste. J. Scott(2003). A Study of the Effects of Affect-based Trust and Cognition-based Trust on Intra-Organizational Knowledge Sharing and Use, available at: www.ProQuest.com.

Iragi, M.(1998). Evaluating the Relation of Job Satisfaction and Staffs' Organizational Commitment in Ahvaz Steel Complex. M.A thesis. Economy and Administrative Sciences Department of Isfahan University.

Khatamianfar, P. (2007). An Evaluation of the Status and Methods of Knowledge Sharing in the Organization of Astan-e-Ghods Razavi 
Library. M.A thesis for Library Science and Information, Mashhad Ferdosi University, Psychology and Educational Science Department, Mashhad.

Lin, W.B. (2007) .The effect of knowledge sharing model "Expert System With Application. Available online at www.siencedirect .com

Mortazavi, A. (2008). The Pathology of Knowledge Sharing in the Organizations: A case Study of an Automotive Manufacturing Company. M.A. thesis. Tehran University. Ghom Higher Education Center.

Nonaka, Ikujiro(1994). A Dynamic Theory of Organizational Knowledge Creation, Organization Science, Vol. 5, No. 1 (Feb., 1994), pp. 14-37.

Nonaka, I. 0., \& Takeuchi, H.(1995). The knowledge creating company. New York: Oxford University Press. pp. 61-68.

Robertson, M. \& O Malley Hammersley, G.(2000), Knowledge Management Practices within a Knowledge-intensive Firm: the Significance of the People Management Dimension, Journal of European Industrial Training , 24, 241-253.

Sarooghi, A. (1996). Organizational Commitment and its Relation with the Tendency to Quit Job. State Managerial Periodical. No.35, pp. 65-73.

Scarbrough , H. \& Carter , C.(2000), Investigating Knowledge Management. CIPD, London.

Skyrme, D.J., (1998), "Knowledge Management: Approaches and Policies", David Skyrme Associates Limited, Highclere, England, 2000.

Skyrme, D. J.(2002) The 3Cs of knowledge sharing: Culture, Competition and Commitment [online].

Swan, Scarborough \& Preston. 1999, "Knowledge management - the next fad to forget people ? " Proceedings of the 7th European Conference on Information Systems, Copenhagen, p.669, pp.5-10.

Wu, Wei-Li; Lin, Chien-Hsin; Hsu, Bi-Fen; Yeh, Ryh-Song (2009). Social Behavior and Personality; ProQuest Psychology Journals pg. 83

Yang ,J.T .\&Wan,C. S.(2004),Advanced organizational Effectiveness and Knowledge Management Implementation ,Tourism Management ,Vol. 25 ,pp. 593-601.

Yosefi, S. Tishevarz Daem, M. Mohammad Kazem, (2010). The Role of the Organizational Commitment in Knowledge Sharing. Police Human Development Periodical, No.30. 